Marcos Felipe Silva de Sá 1

FERnANDA VieIRa RodovalHo-CalleGarl ${ }^{2}$

Maristela Carbol Patta ${ }^{3}$

Patrícia Margareth LizarelLi ${ }^{4}$

Carolina Sales Vieira

Técia Maria Oliveira Maranhão ${ }^{6}$

\section{Macroprolactinemia: as vantagens do rastreamento na prática clínica}

\author{
Advantage of macroprolactinaemia screening in clinical practice
} de Ribeirão Preto da Universidade de São Paulo Avenida Bandeirantes, 3.900 - Campus Universitário CEP 14049-900 - Ribeirão Preto (SP), Brasil Fone: (16) 3602-2804 / Fax: (16) 3633-0946 E-mail: marcosfelipe@fmrp.usp.br
A prolactina (PRL) é um hormônio hipofisário que circula em diferentes formas moleculares: "little" ou "small", monomérica, que é biologicamente ativa (Peso Molecular 23 kDa); "big” (PM 50-60 kDa); "big-big” (PM 150-170 kDa), também conhecida como macroprolactina, que é constituída pela molécula da PRL ligada a um complexo de IgG ${ }^{1,2}$. No soro de indivíduos normais, a forma monomérica é a predominante (85-95\%), enquanto a "big-big" PRL constitui a forma menos frequente $(<1 \%)^{3}$.

A hiperprolactinemia é uma das alterações endócrinas mais comuns do eixo hipotálamohipofisário, acometendo $0,4 \%$ da população adulta e 9-17\% das mulheres com distúrbios reprodutivos. Sua etiologia é variada e, excluindo-se as causas fisiológicas, o uso de algumas medicações e o hipotireoidismo, os tumores hipofisários secretores ou não de PRL representam a principal causa de hiperprolactinemia.

As manifestações clínicas da hiperprolactinemia compreendem uma série de sintomas como os distúrbios menstruais, galactorreia, infertilidade, hirsutismo e alterações neurooftalmológicas, estas últimas quando a causa é um tumor de maiores proporções. A longo prazo, a hiperprolactinemia pode levar à osteoporose como consequência do hipoestrogenismo que se estabelece nessas pacientes.

A associação desses sintomas e sinais com elevação dos níveis de PRL plasmática torna obrigatória, portanto, a investigação da etiologia com foco especial na deteç̧ão de um tumor hipofisário, principalmente os secretores de PRL, chamados prolactinomas, o que é feito sistematicamente através de exames de imagem como a Tomografia Computadorizada (TC) e a Ressonância Nuclear Magnética (RNM) .

Há, entretanto, uma situação clínica que constitui uma verdadeira "armadilha" diagnóstica, na qual uma paciente assintomática ou com sintomatologia pouco expressiva e inespecífica apresenta níveis elevados de PRL plasmática. O aumento da PRL plasmática nesses casos pode ser decorrente de um aumento da sua forma "big-big", a macroprolactina, que é biologicamente pouco ativa, sem repercussões clínicas para o organismo da mulher ${ }^{5}$.

Essa condição é reconhecida há um longo tempo e sua prevalência no universo dos pacientes com hiperprolactinemia parece ser mais elevada do que se pensava. As principais estatísticas publicadas, com dados provenientes de serviços do Canadá, Reino Unido e Irlanda, descrevem taxas de prevalência de 15 a $25 \%$. Estudos realizados em nosso meio

'Professor Titular do Departamento de Ginecologia e Obstetrícia da Faculdade de Medicina de Ribeirão Preto da Universidade de São Paulo - USP - Ribeirão Preto (SP), Brasil.

${ }^{2}$ Professora Adjunta do Departamento de Medicina, Centro de Ciências Biológicas e da Saúde, Universidade Federal de São Carlos - UFSCar - São Carlos (SP), Brasil.

${ }^{3}$ Professora Adjunta do Departamento de Medicina, Centro de Ciências Biológicas e da Saúde, Universidade Federal de São Carlos - UFSCar - São Carlos (SP), Brasil.

${ }^{4}$ Mestre em Tocoginecologia pela Faculdade de Medicina de Ribeirão Preto da Universidade de São Paulo - USP - Ribeirão Preto (SP), Brasil; Médica Ginecologista Obstetra, Clínica Costa Verde, Ubatuba, (SP), Brasil.

${ }^{5}$ Professora do Setor de Reprodução Humana do Departamento de Ginecologia e Obstetrícia da Faculdade de Medicina de Ribeirão Preto da Universidade de São Paulo - USP - Ribeirão Preto (SP), Brasil.

'Professora Titular do Departamento de Tocoginecologia da Universidade Federal do Rio Grande do Norte - UFRN - Natal (RN), Brasil. 
encontraram taxas de $16,5 \%^{6}$ e até a expressiva cifra de $46 \%^{7}$. Esta última, uma exceção, provavelmente reflete o fato de a pesquisa ter sido desenvolvida em um laboratório de referência para dosagens de PRL. Assim, pode-se concluir que a determinação da ocorrência verdadeira de macroprolactinemia é uma tarefa difícil. Outro tópico que tem sido intensamente discutido na literatura se refere à maior prevalência de macroprolactinemia em pacientes com doenças autoimunes. A hiperprolactinemia devido à presença de macroprolactina é reconhecida nas pacientes com lúpus eritematoso sistêmico ${ }^{8}$, mas não parece estar relacionada àquela presente na artrite reumatoide 9 . Essa diferença pode ser consequência do tipo de autoanticorpo circulante, uma vez que na artrite reumatoide a IgM é o tipo de anticorpo predominante ao qual pode não se ligar a PRL.

O dilema imposto pela macroprolactinemia na prática clínica é que, embora ela esteja associada com hiperprolactinemia assintomática, não relacionada à presença de doença hipofisária, sintomas característicos da síndrome hiperprolactinêmica podem estar presentes. Geralmente, essas pacientes apresentam sintomas inespecíficos como: presença de distúrbios menstruais na ausência de galactorreia ou presença de infertilidade na ausência de galactorreia e distúrbios menstruais. No entanto, a associação de galactorreia e distúrbios menstruais poderá ser observada em uma quantidade razoável de pacientes ${ }^{10}$.

Assim, a presença de níveis elevados de PRL associada a esses sintomas levarão fatalmente os clínicos, orientados pelos protocolos clássicos de abordagem dessas pacientes, a solicitarem exames de imagem para descartar a presença de tumores hipofisários e a prescreverem os agonistas dopaminérgicos, além de provavelmente "rotularem" os pacientes como portadores de hiperprolactinemia idiopática, o que pode gerar ansiedade e medo.

Dessa forma, diferenciar a hiperprolactinemia verdadeira (com aumento da forma monomérica) de uma condição clínica aparentemente benigna como a macroprolactinemia torna-se uma conduta prudente e necessária. Muitos pesquisadores estão convencidos de que o rastreamento para macroprolactina deve ser sempre realizado em todos os pacientes com concentrações elevadas de $\mathrm{PRL}^{5,6,11-13}$. Cabe ressaltar que se a paciente apresenta concentrações elevadas de macroprolactina e sintomas clássicos da síndrome hiperprolactinêmica (amenorreia, galactorreia, infertilidade) associados à cefaleia e alterações visuais, o exame de imagem deve ser solicitado. A presença de um resultado de imagem positivo deve ser avaliada com mais cuidado, pois pode corresponder a outros tumores da hipófise que não os prolactinomas ou a erros na análise da imagem ${ }^{10}$. Essa análise evitaria condutas invasivas desnecessárias.

No entanto, para introduzir uma nova metodologia diagnóstica em um protocolo de investigação é imprescindível determinar se o procedimento está disponível, se não é invasivo, se é de fácil execução, de baixo custo e, também, se reduz o custo operacional para o sistema de Saúde, seja ele privado ou público. A cromatografia líquida em coluna de gel (CFG) é considerada o padrão-ouro para detecção de macroprolactina ${ }^{14}$. Porém, é um método muito dispendioso, de difícil execução e de elevado custo, inviável para aplicação na rotina dos laboratórios de investigação clínica. O tratamento do soro hiperprolactinêmico com polietilenoglicol (PEG) é a alternativa de primeira escolha para GFC, pois apresenta a melhor correlação com esse método de referência. O PEG remove as formas macro do plasma a ser analisado. A quantidade de prolactina monomérica existente no sobrenadante é medida no mesmo ensaio, e a taxa de recuperação é calculada com base no valor inicial da amostra. Embora os procedimentos do PEG não estejam totalmente automatizados, trata-se de método reprodutível, de fácil execução e baixo custo disponível na maioria dos laboratórios de bioquímica ${ }^{15}$.

Os benefícios da utilização rotineira do rastreamento para macroprolactina, utilizando a precipitação com PEG, foram demonstrados por Gibney et al. ${ }^{11}$. A prevalência de macroprolactinemia foi $22 \%$ e, nos 12 meses que precederam a introdução do rastreamento, a frequência de solicitação de exames de imagem para pacientes com macroprolactinemia $(73 \%)$ foi semelhante àquela para pacientes com hiperprolactinemia verdadeira (82\%). Alterações consistentes com tumores hipofisários (micro e macroadenomas) foram encontradas predominantemente entre os pacientes com hiperprolactinemia verdadeira (40\%), e entre os portadores de macroprolactinemia em 13\% dos casos, sendo apenas microadenomas. O tratamento com agonistas da DA foi prescrito para $87 \%$ dos pacientes com macroprolactinemia e $82 \%$ dos pacientes com hiperprolactinemia verdadeira. Os autores comentam que a frequência de imagens anormais nos pacientes com macroprolactinemia foi semelhante à encontrada em indivíduos não selecionados, submetidos à exames de imagem da hipófise por outras razões que não a suspeita de doença hipofisária (6-20\%) ou anormalidades hipofisárias encontradas em autópsias $(10-24 \%)^{16}$. Assim, a frequência de alterações da hipófise em indivíduos com macroprolactinemia não é superior à esperada para a população em geral. O não-reconhecimento dessa condição pode levar desnecessariamente à exploração cirúrgica da hipófise, enquanto o diagnóstico de macroprolactinemia permite adotar uma conduta conservadora e mais expectante, mesmo para pacientes que apresentem alterações nos exames de imagem da hipófise com sintomatologia ausente ou leve. 
No estudo citado, o rastreamento de rotina para macroprolactina resultou em um aumento de $27 \%$ nos custos globais com a dosagem de PRL. Em contraste, ocorreu uma redução de 15\% nos custos associados à TC/RNM, evitando-se a realização desses exames nos pacientes com macroprolactinemia identificada, ou seja, em $21 \%$ de todos os indivíduos com hiperprolactinemia. Adicionalmente, ocorreu uma redução de 17\% nos gastos com agonistas dopaminérgicos. Os autores concluíram que o gasto adicional para se realizar o rastreamento para macroprolactina foi compensado pelas economias com exames de imagem desnecessários e tratamentos inadequados.

Em nosso meio, de Soárez et al. ${ }^{12}$ descreveram a frequência e os tipos de testes solicitados após um diagnóstico de concentrações elevadas de PRL e se o diagnóstico de macroprolactinemia resultou em posterior redução dos gastos com exames complementares e tratamento. De Janeiro de 2004 a Abril de 2007, todos os pacientes encaminhados a um centro de diagnóstico privado para dosagem de PRL e que apresentaram concentrações de PRL $>30 \mathrm{ng} / \mathrm{mL}$ foram também avaliados quanto à presença de macroprolactinemia. A conduta clínica indicada para cada paciente frente ao diagnóstico de macroprolactinemia ou hiperprolactinemia verdadeira foi monitorada por um período de seis meses. Do total de 1.793 indivíduos com concentrações de PRL>30 ng/mL, 1.139 (63,5\%) foram classificados como hiperprolactinêmicos verdadeiros e 654 (36,5\%) como macroprolactinêmicos. Os exames de imagem foram realizados em 6,5 e 5,6\% dos pacientes, respectivamente. Em três anos de seguimento, não foram observadas diferenças na frequência da solicitação de exames de imagem. Os macro e microadenomas foram observados em 53,3\% na hiperprolactinemia, enquanto o microadenoma foi observado em 5,4\% dos casos de macroprolactinemia, sem nenhuma ocorrência de macroadenoma. O tratamento dopaminérgico foi instituído em $4 \%$ dos pacientes com hiperprolactinemia e 2,6\% dos pacientes com macroprolactinemia. O custo médio do acompanhamento por paciente no grupo de hiperprolactinêmi$\cos (\mathrm{R} \$ 425,00)$ foi significativamente maior do que no grupo de macroprolactinêmicos $(\mathrm{R} \$ 340,00)$, e o incremento no custo foi de cerca de $25 \%$ para o primeiro grupo. Esses custos incluíram os exames complementares solicitados e a medicação prescrita. Os autores comentam que a diferença entre os grupos foi modesta, muito menor do que esperavam encontrar. É possível que esse resultado tenha sido consequência da persistência da investigação ativa para doenças hipofisárias e tratamento clínico mesmo após o diagnóstico de macroprolactinemia. Mas é mais provável que esses dados possam refletir a pouca familiaridade dos clínicos com a pesquisa da macroprolactina, sua interpretação e a abordagem das pacientes.

Em conclusão, o rastreamento de rotina da macroprolactina em todos os pacientes com hiperprolactinemia é recomendável. Embora a maioria dos laboratórios no país ainda não o esteja realizando de forma rotineira, ele é de baixa complexidade e poderia ser facilmente incorporado às rotinas, não se fazendo necessário o encaminhamento das amostras de sangue para laboratórios de referência mais complexos. Os gestores dos sistemas de Saúde públicos ou privados deveriam encorajar os laboratórios a incorporarem a tecnologia para esse rastreamento e a introduzi-la nos protocolos clínicos para abordagem de pacientes com hiperprolactinemia.

Seria recomendável aos laboratórios que realizam esse exame a uniformização na apresentação dos resultados, fornecendo informações das concentrações de PRL total, das frações monomérica e a forma macro.

Além disso, é preciso difundir entre a comunidade médica os conhecimentos mais atuais sobre esse tema, principalmente no que diz respeito às metodologias diagnósticas disponíveis e o significado clínico das macroprolactinemias. O rastreamento rotineiro da macroprolactina resultará em uma real redução de diagnósticos errôneos, de investigações impróprias e de tratamentos desnecessários, consequentemente acarretando menos gastos para o sistema de Saúde. Possibilitará, ainda, a diminuição da ansiedade e do sofrimento dos pacientes que tenham uma suspeita de tumor hipofisário após um resultado com níveis de PRL elevado, uma vez que o aumento da macroPRL está associado aos pequenos tumores secretores (microadenomas), conforme referências apresentadas acima.

\section{Referências}

1. Carrara HHA, Pinheiro SB, Moura MD, Ferriani RA, Silva de Sá MF. Múltiplas formas moleculares de prolactina em gestantes normais e em portadoras de insuficiência renal crônica. Reprod Clim. 1997;12 Supl 1:13.

2. Sá MFS, Ferriani RA, Moura MD. Modificações nas proporções das três formas moleculares de prolactina secretada por tumores hipofisários. Rev Bras Ginecol Obstet. 1988; 10(6): 1 17-22.

3. Sadideen H, Swaminathan R. Macroprolactin: what is it and what is it importance? Int J Clin Pract. 2006;60(4):457-61.
4. Sá MFS, Pinheiro SB, Trad CS, Moura MD, Ferriani RA. Prolactinoma: avaliação da eficácia dos métodos complementares para seu diagnóstico e da terapia com bromocriptina. Rev Bras Ginecol Obstet. 1992;14(6):272-8.

5. Gibney J, Smith TP, McKenna TJ. Clinical relevance of macroprolactin. Clin Endocrinol (Oxf). 2005;62(6):633-43.

6. Vilar L, Moura E, Canadas V, Gusmão A, Campos R, Leal E, et al. Prevalência da macroprolactinemia entre 115 pacientes com hiperprolactinemia. Arq Bras Endocrinol Metab. 2007;51 (1):86-91. 
7. Hauache OM, Rocha AJ, Maia AC Jr, Maciel RM, Vieira JG. Screening for macroprolactinaemia and pituitary imaging studies. Clin Endocrinol (Oxf). 2002;57(3):327-31.

8. Leaños-Miranda A, Pascoe-Lira D, Chávez-Rueda KA, Blanco-Favela F. Antiprolactin autoantibodies in systemic lupus erythematosus: frequency and correlation with prolactinemia and disease activity. J Rheumatol. $2001 ; 28(7)$ :1546-53.

9. Ram S, Blumberg D, Newton P, Anderson NR, Gama R. Raised serum prolactin in rheumatoid arthritis: genuine or laboratory artefact? Rheumatology (Oxford). 2004;43(10): 1272-4.

10. Vallette-Kasic S, Morange-Ramos I, Selim A, Gunz G, Morange S, Enjalbert A, et al. Macroprolactinemia revisited: a study on 106 patients. J Clin Endocrinol Metab. 2002;87(2):581-8.

11. Gibney J, Smith TP, McKenna TJ. The impact on clinical practice of routine screening for macroprolactin. J Clin Endocrinol Metab. 2005;90(7):3927-32.
12. de Soárez PC, Souza SC, Vieira JG, Ferraz MB. The effect of identifying macroprolactinemia on health-care utilization and costs in patients with elevated serum prolactin levels. Value Health. 2009; $12(6): 930-4$.

13. McKenna TJ. Should macroprolactin be measured in all hyperprolactinaemic sera? Clin Endocrinol (Oxf). 2009;71(4):466-9.

14. Reis RM, Ribeiro CT, Sá MFS, Carrara HHA, Machado HR, Santos AC. Macroprolactinoma resistente aos agonistas dopaminérgicos: relato de caso. Rev Bras Ginecol Obstet. 2004;26(8):663-7.

15. Kavanagh L, McKenna TJ, Fahie-Wilson MN, Gibney J, Smith TP. Specificity and clinical utility of methods for the detection of macroprolactin. Clin Chem. 2006;52(7):1366-72.

16. Molitch ME, Russell EJ. The pituitary "incidentaloma". Ann Intern Med. 1990;112(12):925-31. 